BMJ

Open

Gastroenterology

\title{
Autoantibodies in chronic hepatitis $C$ virus infection: impact on clinical outcomes and extrahepatic manifestations
}

\author{
Andrew J Gilman, ${ }^{1}$ An $\mathrm{K} \mathrm{Le},{ }^{2}$ Changqing Zhao, ${ }^{3}$ Joseph Hoang, ${ }^{2}$ Lee A Yasukawa, ${ }^{4}$ \\ Susan C Weber, ${ }^{4}$ John M Vierling, ${ }^{5}$ Mindie H Nguyen ${ }^{2}$
}

To cite: Gilman AJ, Le AK, Zhao C, et al. Autoantibodies in chronic hepatitis C virus infection: impact on clinical outcomes and extrahepatic manifestations. BMJ Open Gastro 2018;5:e000203. doi:10.1136/ bmjgast-2018-000203

Received 24 February 2018 Revised 27 March 2018 Accepted 2 April 2018
Check for updates

${ }^{1}$ Department of Medicine, Stanford University, Palo Alto, California, USA

${ }^{2}$ Division of Gastroenterology and Hepatology, Stanford University, Palo Alto, California, USA

${ }^{3}$ Department of Cirrhosis, Institute of Liver Disease, Shuguang Hospital, ShangHai, China

${ }^{4}$ Center for Clinical Informatics, Stanford University, Stanford, California, USA

${ }^{5}$ Departments of Medicine and Surgery, Baylor College of Medicine, Houston, Texas, USA

Correspondence to Dr Mindie H Nguyen; mindiehn@stanford.edu

\section{ABSTRACT}

Goals To examine the role that autoantibodies (autoabs) play in chronic hepatitis $\mathrm{C}$ virus (HCV) regarding demographics, presence of extrahepatic manifestations and long-term outcomes in a large US cohort.

Background Auto-abs have been reported to be prevalent in patients with chronic HCV infection, but data on the natural history of these patients are limited. Study The study included 1556 consecutive patients with HCV without concurrent HIV and/or HBV who had testing for antinuclear antibody (ANA), antimitochondrial antibody (AMA), antismooth muscle antibody (ASMA) and/ or antiliver kidney microsomal antibody (LKM). Primary outcomes included development of cirrhosis, hepatic decompensations, hepatocellular carcinoma (HCC), mortality and/or sustained virological response (SVR) to antiviral therapy.

Results A total of 388 patients tested positive for any auto-ab (ANA 21.8\%, ASMA 13.3\%, AMA 2.2\% and LKM $1.2 \%)$. Patients who tested positive versus negative were more likely to be women $(29.3 \%$ vs $20.9 \%, p<0.001)$ and less likely to achieve SVR with most treated patients receiving interferon-based therapies $(37.2 \%$ vs $47.1 \%$, $p=0.031$ ). There was no difference between groups for baseline laboratory data, disease state or rate of extrahepatic manifestations ( $42.8 \%$ vs $45.0 \%, p=0.44$ ). Kaplan-Meier analysis revealed no statistically significant difference between groups for the 10-year development of cirrhosis, hepatic decompensations, HCC nor survival. Furthermore, auto-ab positivity was only found to be a predictor for a lower rate of SVR on multivariate analysis (adjusted OR=1.61, $95 \% \mathrm{Cl} 1.00$ to 2.58, $\mathrm{p}=0.048$ ).

Conclusions In our cohort, auto-ab positivity was common, especially in women, and predicted a lower rate of SVR but otherwise had no impact on the natural history of chronic HCV or presence of extrahepatic manifestations.

\section{INTRODUCTION}

Hepatitis $\mathrm{G}$ virus (HCV) infection is a major public health problem with chronic HCV infection (CHC) afflicting an estimated 71 million people globally and 2.7-4.1 million people in the USA. ${ }^{2}$ There is marked variation in prevalence both within the USA and
Summary box

What is already known about this subject?

- Autoantibodies (auto-abs) have been reported to be prevalent in patients with chronic hepatitis $\mathrm{C}$ virus (HCV) infection, but data on the natural history of these patients are limited.

- Furthermore, available studies have a limited number of patients and focus on differences in demographics.

What are the new findings?

- Our study focused on long-term clinical outcomes in a large, diverse cohort demonstrating that auto-ab positivity played no role in the progression of liver disease in patients with chronic HCV, including development of cirrhosis, hepatic decompensations, hepatocellular carcinoma (HCC) nor mortality. However, auto-ab positivity was associated with a decreased achievement of sustained virological response (SVR).

How might it impact on clinical practice in the foreseeable future?

- Auto-ab positivity in the setting of chronic HCV should not prompt worse prognostication in similar patients nor should auto-abs be routinely checked.

- However, high-efficacy direct acting antiviral treatment should have additional importance, as auto-ab-positive patients are less likely to achieve SVR with interferon-based therapies.

abroad, reflecting diversity of the geographic populations, as well as resources for prevention and treatment. ${ }^{1}$ CHC has previously been associated with the presence of autoantibodies (auto-abs), including antinuclear antibody (ANA), antismooth muscle antibody (ASMA), antimitochondrial antibody (AMA), antiliver kidney microsomal antibody (LKM) and antisoluble liver antigen (SLA) which are also present in autoimmune liver diseases. ${ }^{3-19}$

The significance of auto-ab positivity in the natural history of $\mathrm{CHC}$, and whether 
their presence reflects a separate disease entity entirely, remains unclear. Questions remain whether the presence of auto-abs could represent an enhanced propensity for an inflammatory response to the virus and whether this yields a resulting higher incidence of long-term complications of or extrahepatic manifestations from infection. Multiple studies have reported conflicting results regarding differences in demographic factors (such as sex, age and race or ethnicity) and/or clinical outcomes (such as sustained virological response (SVR), stage of fibrosis and documentation of cirrhosis) between patients infected with HCV with and without auto-abs..$^{8-11} 1314$ 16-25 Four studies demonstrated a higher prevalence of auto-ab positivity in women. ${ }^{10} 192122$ Only two studies demonstrated decreased rates of SVR in auto-ab-positive patients, ${ }^{26} 27$ while 11 studies found no difference in SVR rates. ${ }^{8} 10111314181923-2528$ Furthermore, the majority of studies have come from Asia, Europe, Africa and South America. Only two published studies were from the USA, one of which focused on HCV/HIV coinfection and the relationship with auto-ab prevalence, ${ }^{20}$ and the other focused on the relationship between the prevalence of auto-abs and virological response to interferon (IFN). ${ }^{8}$ To the best of our knowledge, no studies have analysed the impact of the presence or absence of auto-abs and the subsequent development of cirrhosis, hepatocellular carcinoma (HCC) or mortality in a longitudinal fashion. The aim of this study was to characterise the differences in clinical presentation and outcomes on longitudinal follow-up between patients with CHC with and without auto-ab positivity in a large, ethnically diverse population of patients in the USA.

\section{MATERIALS AND METHODS Study design and study patients}

We performed a retrospective cohort study of consecutive patients with CHC who were seen at Stanford Health Care in Palo Alto, California between January 1997 and August 2016 and had auto-ab testing. Patients were initially identified via an International Classification of Diseases, Ninth Revision (ICD-9) or International Classification of Diseases, Tenth Revision (ICD-10) code query with a subsequent review of their electronic medical record for confirmation of CHC diagnosis and data abstraction using a case report form. Inclusion criteria consisted of: (1) presence of CHC (as determined by positive anti-HCV antibody and detectable HCV RNA using PCR or documented CHC in provider notes) and (2) presence of auto-ab testing (ie, ANA, ASMA, AMA, LKM and/or SLA). Patients with hepatitis B virus (HBV) or HIV coinfections were excluded.

Auto-ab tests for ANA, ASMA, AMA, LKM and/or SLA were considered positive either at the time of initial presentation to Stanford Health Care or at any time during follow-up. Study index date was defined as the date of auto-ab testing. Titre positivity, performed by serial dilutions, was defined by standard cut-off values for the Stanford Health Care laboratory: ANA $\geq 1: 40$ and ASMA $\geq 1: 20$. For subgroup analyses, a high titre was defined as ANA or ASMA $\geq 1: 160$. AMA positivity, performed by M2 enzyme immunoassay at Mayo Medical Laboratories, was defined by their cut-off value of $\geq 1.0$ Units. LKM positivity, performed by semi-quantitative ELISA at ARUP laboratories, was defined by their cut-off value of $\geq 25.0$ Units. For laboratory data obtained from referring facilities, positivity was defined according to the facility's standard cutoffs. When a referring facility's objective laboratory data were not available, patients were considered auto-ab positive if positivity was documented in the provider notes.

Patient race or ethnicity was determined by self-reporting and/or country of emigration. Race or ethnicity was categorised as Caucasian, Asian, Black or Hispanic.

The presence of comorbidities of hypertension (HTN), diabetes mellitus (DM), coronary artery disease (CAD), chronic obstructive pulmonary disease (COPD) and chronic kidney disease (CKD) was determined by querying ICD codes or documentation in provider notes. Similarly, the presence of extrahepatic disease manifestations of mixed cryoglobulinaemia, depression, Sjögren's syndrome, rheumatoid arthritis (RA), non-Hodgkin's B-cell lymphoma, DM and CKD was determined by ICD code query or documentation in provider notes.

SVR was defined as the absence of detectable HCV RNA at 12 or 24 weeks after treatment completion using an assay with a cut-off of $\leq 15 \mathrm{IU} / \mathrm{mL}$. The Model for End-Stage Liver Disease (MELD) and Child-Turcotte-Pugh (CTP) scores were used to assess 90-day mortality estimates and hepatic function and complications of portal hypertension, respectively, in patients with cirrhosis.

This study was approved by the Institutional Review Board of Stanford University, Stanford, California, USA.

\section{Primary outcomes}

The presence of cirrhosis was determined at baseline and during follow-up based on the presence of stage 4 fibrosis on liver biopsy, clinical hepatic decompensation (jaundice, encephalopathy, ascites and variceal gastrointestinal bleeding) or other radiologic, endoscopic or laboratory findings such as the presence of a nodular liver on imaging, thrombocytopaenia $(<120000$ per $\mu \mathrm{L})$ or intra-abdominal portal venous collaterals. Similarly, the presence of HCC was determined based on cytology, tissue pathology or non-invasive criteria recommended by the American Association for the Study of Liver Diseases. ${ }^{29}$ Cirrhosis or HCC diagnosed within 6 months of initial presentation to our institution was considered to be prevalent cases. Mortality was determined via a chart review or via a search of the National Death Index that was performed for patients seen before 1 January 2015 who were not known to be deceased.

\section{Statistical analysis}

Continuous variables were categorised as a mean $\pm \mathrm{SD}$ and statistically compared using Student's t-test when a 
Table 1 Autoantibody positivity distribution among all patients

\begin{tabular}{|c|c|c|c|c|c|c|c|c|c|}
\hline & $\begin{array}{l}\text { Overall } \\
(n=1556)\end{array}$ & $\begin{array}{l}\text { Male } \\
(n=813)\end{array}$ & $\begin{array}{l}\text { Female } \\
(n=743)\end{array}$ & $P$ value & $\begin{array}{l}\text { White } \\
(n=827)\end{array}$ & $\begin{array}{l}\text { Asian } \\
(n=177)\end{array}$ & $\begin{array}{l}\text { Black } \\
(n=122)\end{array}$ & $\begin{array}{l}\text { Hispanic } \\
(n=277)\end{array}$ & $P$ value \\
\hline ANA $(n=1461)$ & $21.8 \%$ & $18.1 \%$ & $25.9 \%$ & $<0.001$ & $20.2 \%$ & $21.4 \%$ & $28.3 \%$ & $20.8 \%$ & 0.30 \\
\hline ASMA $(n=483)$ & $13.3 \%$ & $11.9 \%$ & $14.6 \%$ & 0.38 & $11.4 \%$ & $16.1 \%$ & $10.5 \%$ & $18.4 \%$ & 0.30 \\
\hline AMA $(n=505)$ & $2.2 \%$ & $2.4 \%$ & $2.0 \%$ & 0.78 & $1.2 \%$ & $5.8 \%$ & $2.4 \%$ & $1.8 \%$ & 0.19 \\
\hline LKM (n=86) & $1.2 \%$ & $0 \%$ & $2.0 \%$ & 0.39 & $2.1 \%$ & $0 \%$ & $0 \%$ & $0 \%$ & 0.86 \\
\hline
\end{tabular}

ANA, antinuclear antibody; AMA, antimitochondrial antibody; ASMA, antismooth muscle antibody; LKM, anti-liver kidney microsomal antibody.

normal distribution was observed. Otherwise, non-parametric methods were applied. Categorical variables were expressed as proportions (\%) and analysed using the $\chi^{2}$ test. Kaplan-Meier analyses were utilised to determine the cumulative incidence of cirrhosis, decompensation events, HCC and mortality with comparisons between patients with and without auto-ab positivity performed using the log-rank test. Univariate Cox proportional hazards models were utilised to estimate HR and 95\% CIs relating various factors, such as race and ethnicity, auto-ab positivity and comorbidities, to the incidence of SVR, cirrhosis, HCC and mortality. Stepwise multivariate models were constructed to adjust for age, gender and other clinically relevant and/or statistically significant factors on univariate analysis $(\mathrm{p}<0.10)$. Statistical significance was defined as a two-sided $p$ value $\leq 0.05$. All statistical analyses were performed using Stata V.14.2 (Stata Corporation, College Station, Texas, USA).

\section{RESULTS}

Antibody prevalence and baseline characteristics

A total of 1556 consecutive patients with CHC who also had testing for any auto-ab were included in the study. The database of our patient with CHC includes a total of 9351 patients, of which only 1678 had auto-ab testing of any kind with 122 of those patients excluded from this study due to HIV and/or HBV coinfections. Of the remaining 1556 patients, 388 tested positive for any auto$\mathrm{ab}$, with the distribution among auto-abs displayed in table 1. Data related to titres and the pattern of immunofluorescence are shown in table 2. The distribution of antiviral treatment regimens was not different between auto-ab-positive and auto-ab-negative patients, with IFN-based therapies being the most commonly used (86.6\% and $84.4 \%$, respectively).

Baseline characteristics, comorbidities, extrahepatic manifestations, treatment status, HCV genotype and disease severity for all study participants are outlined in table 3. For items with data that were not available for all patients, the number of patients with data available is designated in the first column for the two subsequent columns, respectively. Female patients were more likely to test positive for auto-abs $(29.3 \%$ vs $20.9 \%, \mathrm{p}<0.001)$ than male patients. There was no difference among ethnicities for auto-ab presence $(\mathrm{p}=0.11)$. Furthermore, there was no difference between auto-ab-positive and auto-ab-negative groups in the proportions of patients having $\geq 2$ comorbidities $(24.2 \%$ vs $28.0 \%, \mathrm{p}=0.15$ ) or extrahepatic manifestations of HCV infection (42.8\% vs $45.0 \%, \mathrm{p}=0.44)$. The distribution of HCV genotype $(\mathrm{p}=0.48)$, baseline MELD ( $\mathrm{p}=0.83)$ and baseline Child's class $(p=0.24)$ was also similar between auto-ab-positive and auto-ab-negative groups. Interestingly, auto-ab-positive patients were significantly less likely to achieve SVR than patients without auto-abs overall $(37.2 \%$ vs $47.1 \%$, $\mathrm{p}=0.031$ ). However, when stratifying patients by IFN-based versus IFN-free therapy received, SVR was lower for auto-ab-positive patients treated with IFN-based therapy

Table 2 Titre and pattern distribution

\begin{tabular}{lrlrll}
\hline ASMA titre & & ANA titre & & ANA pattern & \\
\hline $1: 20$ & $82.1 \%$ & $1: 40$ & $23.2 \%$ & Homogeneous & $29.3 \%$ \\
$1: 40$ & $7.4 \%$ & $1: 80$ & $22.9 \%$ & Speckled & $41.0 \%$ \\
$1: 80$ & $6.3 \%$ & $1: 160$ & $27.5 \%$ & Nucleolar & $3.3 \%$ \\
$1: 160$ & $2.1 \%$ & $1: 320$ & $8.8 \%$ & Centromere & $0.3 \%$ \\
$1: 320$ & $1.1 \%$ & $1: 640$ & $8.5 \%$ & Cytoplasmic mitochondrial & $5.3 \%$ \\
$1: 1280$ & $1.1 \%$ & $1: 40-1: 160$ & $3.3 \%$ & Homogeneous and speckled & $0.3 \%$ \\
& & $1: 40-1: 640$ & $1.0 \%$ & Homogeneous, speckled and mitotic spindle & $0.7 \%$ \\
& & $1: 1280$ & $4.3 \%$ & Homogeneous and nucleolar & $0.3 \%$ \\
\hline
\end{tabular}

ANA, antinuclear antibody; ASMA, antismooth muscle antibody. 
Table 3 Baseline characteristics

\begin{tabular}{|c|c|c|c|}
\hline & $\begin{array}{l}\text { Autoantibody testing positive } \\
(\mathrm{n}=388)\end{array}$ & $\begin{array}{l}\text { Autoantibody testing } \\
\text { negative }(n=1168)\end{array}$ & $P$ value \\
\hline \multicolumn{4}{|l|}{ Sex (column \% (row \%)) } \\
\hline Male & $43.8 \%(20.9 \%)$ & $55.1 \%(79.1 \%)$ & \multirow[t]{2}{*}{$<0.001$} \\
\hline Female & $56.2 \%(29.3 \%)$ & $45.0 \%(70.7 \%)$ & \\
\hline Age (years) & $51.5 \pm 10.8$ & $52.3 \pm 10.5$ & 0.21 \\
\hline BMI (n=208/730) & $28.7 \pm 7.5$ & $28.3 \pm 6.7$ & 0.49 \\
\hline History of tobacco use $(n=194 / 675)$ & $45.4 \%$ & $51.9 \%$ & 0.11 \\
\hline History of alcohol use $(n=147 / 531)$ & $23.1 \%$ & $27.9 \%$ & 0.25 \\
\hline \multicolumn{4}{|l|}{ Race (column \% (row \%)) } \\
\hline White & $54.3 \%(22.3 \%)$ & $61.0 \%(77.8 \%)$ & \multirow{4}{*}{0.11} \\
\hline Asian & $14.2 \%(27.1 \%)$ & $12.2 \%(72.9 \%)$ & \\
\hline Black & $10.3 \%(31.3 \%)$ & $7.3 \%(68.8 \%)$ & \\
\hline Hispanic Ethnicity & $21.2 \%(26.0 \%)$ & $19.5 \%(74.0 \%)$ & \\
\hline \multicolumn{4}{|l|}{ Comorbidities } \\
\hline$\geq 2$ Comorbidities & $24.2 \%$ & $28.0 \%$ & 0.15 \\
\hline Hypertension & $38.9 \%$ & $39.8 \%$ & 0.76 \\
\hline Diabetes mellitus & $21.4 \%$ & $21.9 \%$ & 0.83 \\
\hline Coronary artery disease & $10.3 \%$ & $12.7 \%$ & 0.22 \\
\hline Chronic obstructive pulmonary disease & $3.4 \%$ & $4.5 \%$ & 0.32 \\
\hline Chronic kidney disease & $11.9 \%$ & $14.7 \%$ & 0.16 \\
\hline \multicolumn{4}{|l|}{ Extrahepatic manifestations } \\
\hline Any extrahepatic manifestation & $42.8 \%$ & $45.0 \%$ & 0.44 \\
\hline Mixed cryoglobulinaemia & $2.3 \%$ & $1.9 \%$ & 0.59 \\
\hline Depression & $21.4 \%$ & $24.7 \%$ & 0.19 \\
\hline Sjögren's syndrome & $0.8 \%$ & $0.3 \%$ & 0.22 \\
\hline Rheumatoid arthritis & $3.4 \%$ & $3.0 \%$ & 0.73 \\
\hline Lymphoma & $1.0 \%$ & $0.4 \%$ & 0.18 \\
\hline \multicolumn{4}{|l|}{ Treatment status } \\
\hline $\begin{array}{l}\text { History of HCV antiviral therapy prior to } \\
\text { presentation }(n=284 / 679)\end{array}$ & $32.0 \%$ & $36.1 \%$ & 0.23 \\
\hline Received antiviral therapy after presentation & $28.6 \%$ & $30.6 \%$ & 0.47 \\
\hline Received antiviral therapy at any time & $43.3 \%(168)$ & $42.3 \%(494)$ & 0.73 \\
\hline SVR achieved $(n=149 / 452)$ & $37.2 \%$ & $47.1 \%$ & 0.031 \\
\hline IFN-based therapy & $29.0 \%$ & $42.7 \%$ & 0.012 \\
\hline DAA therapy & $67.6 \%$ & $71.4 \%$ & 0.65 \\
\hline \multicolumn{4}{|l|}{ HCV genotype $(n=281 / 773)$} \\
\hline 1 Untyped & $15.7 \%$ & $11.3 \%$ & \multirow{7}{*}{0.48} \\
\hline $1 \mathrm{a}$ & $34.5 \%$ & $36.4 \%$ & \\
\hline $1 b$ & $21.0 \%$ & $21.9 \%$ & \\
\hline 2 & $13.9 \%$ & $14.6 \%$ & \\
\hline 3 & $12.5 \%$ & $12.2 \%$ & \\
\hline 4 & $0.7 \%$ & $1.9 \%$ & \\
\hline 6 & $1.8 \%$ & $1.8 \%$ & \\
\hline \multicolumn{4}{|l|}{ Baseline disease state and severity } \\
\hline Baseline cirrhosis & $41.5 \%$ & $44.3 \%$ & 0.34 \\
\hline
\end{tabular}


Table 3 Continued

\begin{tabular}{|c|c|c|c|}
\hline & $\begin{array}{l}\text { Autoantibody testing positive } \\
(n=388)\end{array}$ & $\begin{array}{l}\text { Autoantibody testing } \\
\text { negative }(n=1168)\end{array}$ & P value \\
\hline Baseline history of decompensation & $28.4 \%$ & $29.7 \%$ & 0.61 \\
\hline Baseline HCC & $4.1 \%$ & $4.9 \%$ & 0.54 \\
\hline $\log _{10}$ HCV RNA (IU/mL) $(n=263 / 777)$ & $4.58 \pm 0.02$ & $4.59 \pm 0.02$ & 0.0016 \\
\hline $\begin{array}{l}\text { Baseline MELD in patients with baseline } \\
\text { cirrhosis }\end{array}$ & $16.3 \pm 7.9$ & $16.6 \pm 8.6$ & 0.83 \\
\hline \multicolumn{4}{|l|}{ Baseline CTP ( $n=113$ /408) } \\
\hline$A$ & $36.3 \%$ & $40.2 \%$ & \\
\hline $\mathrm{B}$ & $35.4 \%$ & $39.0 \%$ & 0.24 \\
\hline $\mathrm{C}$ & $28.3 \%$ & $20.8 \%$ & \\
\hline
\end{tabular}

BMI, body mass index; CTP, Child-Turcotte-Pugh; DAA, direct acting antiviral; HCC, hepatocellular carcinoma; HCV, hepatitis C virus; IFN, interferon; MELD, Model for End-Stage Liver Disease; SVR, sustained virological response.

(29.0\% vs $42.7 \%, \mathrm{p}=0.012)$ but not those treated with IFN-free direct acting antiviral (DAA) therapy $(67.5 \%$ vs $71.4 \%, \mathrm{p}=0.65)$.

No significant differences between the auto-ab-positive and auto-ab-negative groups were observed for any of the recorded baseline laboratory data.

\section{Incidence of primary outcomes}

Despite the differences outlined above, Kaplan-Meier analysis revealed no statistically significant difference between auto-ab-positive and auto-ab-negative patients for the development of cirrhosis, hepatic decompensations, HCC

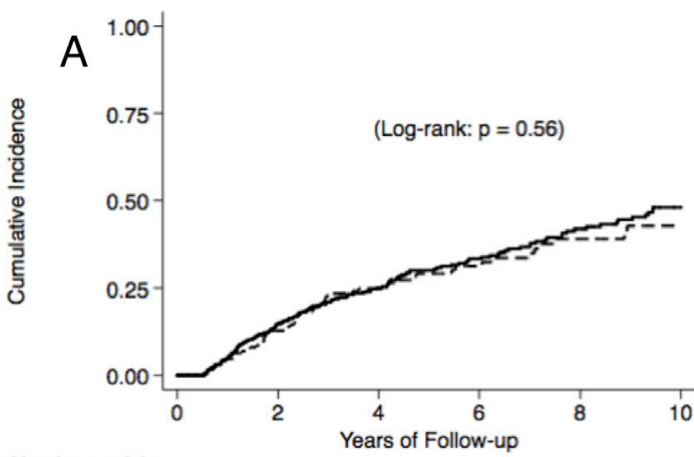

Number at risk
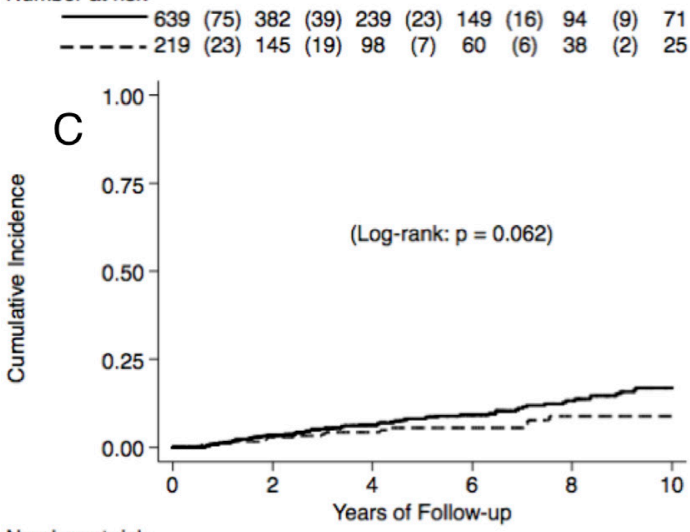

Number at risk

${ }_{-1093(29)} 713$ (18) 448 (12) 290 (10) 187 (7) 142

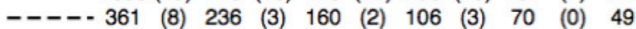

and survival (figure 1A-D). Similar data were obtained on subgroup analyses including only patients with hightitre auto-abs (ANA and/or ASMA $\geq 1: 160$ ) compared with patients without auto-abs. For this subgroup analysis, there was no statistically significant difference at 5 -year $\left(28.59 \%\right.$ vs $\left.30.02 \%, \mathrm{p}_{5}=0.64\right)$ or 10 -year incidence of cirrhosis $\left(38.93 \%\right.$ vs $\left.48.02 \%, \mathrm{p}_{10}=0.36\right)$, hepatic decompensations (5-year incidence: $35.85 \%$ vs $43.94 \%, \mathrm{p}_{5}=0.19$; 10-year incidence: $71.28 \%$ vs $68.24 \%, \mathrm{p}_{10}=0.74$ ), nor survival (5-year incidence: $73.32 \%$ vs $75.09 \%, \mathrm{p}_{5}=0.57$; 10-year incidence: $56.70 \%$ vs $\left.58.67 \%, \mathrm{p}_{10}=0.45\right)$. However,

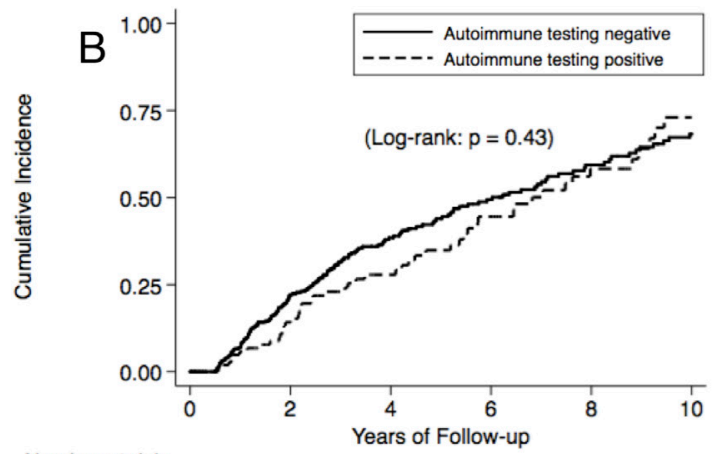

Number at risk

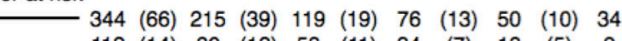

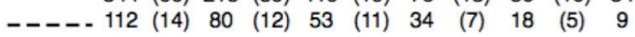

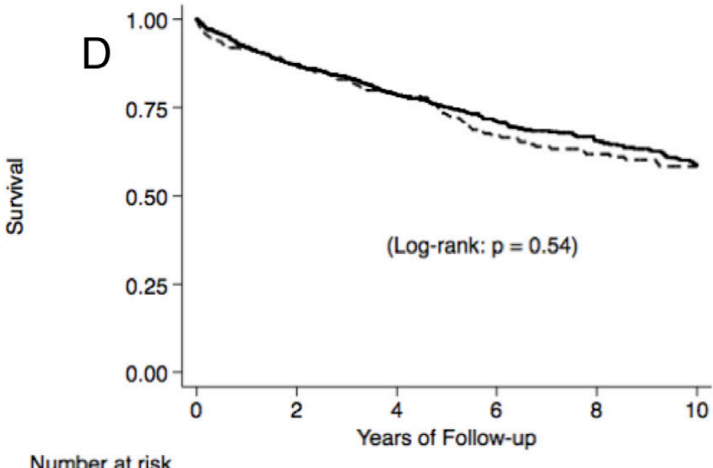

Number at risk

$1155(129) 762(64) 499$ (41) 333 (22) 222 (20) 157 - - - - 379 (46) 254 (21) 181 (23) 118 (8) 81 (4) 56

Figure 1 10-year incidence of primary outcomes in patients with versus without any auto-ab positivity: (A) cirrhosis, (B) hepatic decompensations, (C) HCC and (D) survival. auto-abs, autoantibodies; HCC, hepatocellular carcinoma. 
Table 4 Multivariate analysis of auto-ab positivity

Multivariate HR $(95 \% \mathrm{Cl})$

$P$ value

\begin{tabular}{lll}
\hline Cirrhosis $^{*}$ & $0.84(0.54$ to 1.30$)$ & 0.43 \\
Hepatic decompensations* & $0.61(0.14$ to 2.72$)$ & 0.51 \\
HCC $\dagger$ & $0.83(0.42$ to 1.66$)$ & 0.60 \\
Mortality $\dagger$ & $1.22(0.79$ to 1.87$)$ & 0.37 \\
\hline
\end{tabular}

\begin{tabular}{lll}
\hline & $\begin{array}{l}\text { Adjusted OR } \\
(\mathbf{9 5 \%} \mathbf{C l})\end{array}$ & P value \\
\hline Treatment without SVR $\ddagger$ & $1.61(1.00$ to 2.58$)$ & 0.048 \\
\hline
\end{tabular}

${ }^{*}$ Controlled for: age, gender, $\geq 2$ comorbidities, history of tobacco use, ethnicity and treatment without SVR.

†Controlled for the above as well as cirrhosis.

$\ddagger$ Controlled for: cirrhosis, prior treatment failure, age, gender and virological regimen used.

auto-ab, autoantibody; HCC, hepatocellular carcinoma;

SVR, sustained virological response.

a significantly lower incidence of HCC was observed for the high-titre auto-ab group compared with the group without any auto-abs (5-year incidence: $2.19 \%$ vs $8.16 \%$, $\mathrm{p}_{5}=0.041 ; 10$ year: $6.85 \%$ vs $\left.16.79 \%, \mathrm{p}_{10}=0.031\right)$. There were only four cases of HCC of the 140 high-titre patients at risk in the high-titre patient group.

\section{Multivariate analysis}

Auto-ab positivity was neither a predictor for the development of cirrhosis, hepatic decompensations, HCC, nor for mortality when controlled for age, sex, $\geq 2$ comorbidities, history of tobacco use, ethnicity, and treatment and SVR status (table 4). These findings were also observed on subgroup analysis of high-titre patients as compared with patients without auto-abs. High-titre auto-abs were not significant predictors of cirrhosis (adjusted $\mathrm{OR}=0.78$, $95 \%$ CI 0.28 to $2.17, \mathrm{p}=0.63$ ), hepatic decompensations (adjusted $\mathrm{OR}=0.88,95 \%$ CI 0.34 to $2.25, \mathrm{p}=0.79$ ), HCC (adjusted OR=0.50, 95\% CI 0.07 to $3.77, \mathrm{p}=0.50$ ), nor mortality (adjusted OR $=1.43$, 95\% CI 0.42 to 4.87 , $\mathrm{p}=0.57)$. As expected, lack of SVR and older age were both predictors of development of cirrhosis $(6.14,95 \%$ CI 2.96 to $12.73, \mathrm{p}<0.001 ; 1.19,95 \%$ CI 1.08 to 1.32 , $\mathrm{p}=0.001)$, HCC $(27.98,95 \%$ CI 3.86 to $202.59, \mathrm{p}=0.001$; $1.30,95 \%$ CI 1.13 to $1.50, \mathrm{p}<0.001)$ and mortality (23.80, $95 \%$ CI 5.87 to $96.49, \mathrm{p}<0.001 ; 1.35,95 \%$ CI 1.22 to 1.48 , $\mathrm{p}<0.001)$. Having $\geq 2$ comorbidities did not predict the development of cirrhosis $(1.43,95 \%$ CI 0.98 to 2.09, $\mathrm{p}=0.062$ ).

Interestingly, auto-ab positivity was predictive of failure to achieve SVR with treatment on univariate analysis (unadjusted $\mathrm{OR}=1.59,95 \% \mathrm{CI} 1.10$ to $2.38, \mathrm{p}=0.013$ ) and on multivariate analysis (adjusted $\mathrm{OR}=1.61,95 \%$ CI 1.00 to $2.58, \mathrm{p}=0.048$ ) after controlling for cirrhosis, treatment and SVR status, age, sex and antiviral regimen (table 4). This finding was not observed on subgroup analysis for high-titre patients. High-titre auto-abs were a significant predictor of failure to achieve SVR on univariate analysis (unadjusted $\mathrm{OR}=1.79,95 \%$ CI 1.05 to $3.06, \mathrm{p}=0.034$ ) but this was not significant on multivariate analysis (adjusted $\mathrm{OR}=1.71,95 \%$ CI 0.90 to $3.23, \mathrm{p}=0.10$ ) after controlling for the same variables as above. As expected, the presence of cirrhosis predicted failure to achieve SVR on both univariate (unadjusted $\mathrm{OR}=1.22$, 95\% CI 1.04 to 1.43 , $\mathrm{p}=0.014$ ) and multivariate analyses (adjusted $\mathrm{OR}=1.98$, $95 \%$ CI 1.30 to $3.01, p=0.001$ ).

\section{DISCUSSION}

In our cohort consisting of 1556 ethnically diverse patients with CHC at a large, tertiary care, academic centre, we found an overall auto-ab prevalence of $24.9 \%$ with a wide array of various antibodies, patterns and titres. The diversity, size and length of follow-up of our cohort support that this study is the most comprehensive analysis of the phenomenon of auto-ab presence in $\mathrm{CHC}$ and its effect on clinical outcomes to date. We found that female patients were more likely to have auto-abs, ethnicity played no role in the likelihood of their presence and they did not change the natural history of CHC. This is observed despite the role that auto-ab's presence played in virological failure in response to antiviral therapy.

In addition, subgroup analysis that compared only high-titre (ANA or ASMA $\geq 1: 160$ ) patients to those without auto-abs generally demonstrated the same results. Though there was a significantly lower incidence of HCC in the high-titre patients, there were only four HCC cases among the 140 patients at risk in both groups and a high titre was not found to predict HCC on multivariate analysis. This further supports that auto-ab positivity, even at very high titres, does not change the natural history of CHC. Interestingly, a high titre of ANA ( $49.8 \%$ of patients with any ANA positivity) was much more frequent in our cohort than a high titre of ASMA (4.3\% of patients with any ASMA positivity). Perhaps this represents a greater degree of similarity between the molecular structure of HCV and ANA than ASMA, resulting in a more potent stimulator to development of auto-abs, as has been suggested in other studies. ${ }^{30}$

Regarding the overall prevalence of auto-abs in $\mathrm{CHC}$, previous studies have had mixed results, small study populations and various geographic locations that introduce questions regarding external validity. One study in Europe found differing prevalence at three centres across that continent. ${ }^{19}$ To our knowledge, only two studies have been performed with patients in the USA, both with small sample sizes, the largest being $170 .{ }^{820}$ One of these included HIV coinfected individuals in their analysis. ${ }^{20}$ Our data provide a more complete analysis of the prevalence of auto-abs in CHC for US patients.

Additionally, the clinical management of $\mathrm{CHC}$ has changed dramatically in recent years as new IFN-free regimens became available. One consistent question among previous studies is the effect that auto-abs may have on treatment response, a question that has had mixed answers. Only two studies shared our findings of 
a decreased SVR among auto-ab-positive patients, ${ }^{26} 27$ while 11 others found no difference. $810111314181923-2528$ The two positive studies were published in 2012 and 1997 respectively, while the negative studies were published between 1994 and 2013, with nine of them being published before 2010. Our large population with longterm follow-up, wide distribution of auto-ab titres and patterns, US location and diverse virological regimens utilised demonstrates that there is no significant impact that the presence of auto-abs has on patients with $\mathrm{CHC}$, except a statistically and clinically significant lower chance of achieving SVR (9.9\% absolute risk increase). However, the most common virological regimen used in our population was IFN-based, and this difference in achievement of SVR was not observed in those patients who received IFN-free direct acting antiviral regimens. Additionally, it remains unclear if achieving SVR results in a change in titres, either increase or decrease, a question that could not be answered in the current study due to lack of post-SVR follow-up data.

Furthermore, we found no evidence that patients with auto-abs were more likely to develop extrahepatic manifestations of their disease, reinforcing the concept that auto-abs in CHC may be a laboratory phenomenon rather than a different phenotypic expression of CHC. Alternatively, another perspective is that the auto-abs in $\mathrm{CHC}$ might not reflect a true autoimmunity potential or represent a surrogate for an additive pathogenic mechanism. Instead, their presence may be dictated by the systemic immune response to chronic apoptotic destruction of hepatocytes. This would fit with the high frequency of ANA seen in patients with acute alcoholic hepatitis and acute viral hepatitis that resolves spontaneously. ${ }^{31}$ Additionally, it has been previously postulated that molecular mimicry and similarities between HCV antigens and host antigens are partly responsible for the development of ANA and ASMA. ${ }^{30}$ Despite the lack of differences in the prevalence of extrahepatic diseases between auto-ab-positive and auto-ab-negative patients in our cohort, we did observe a high prevalence of these manifestations overall. For our patients with auto-abs, the most common manifestations were DM (21.4\%), depression (21.4\%), CKD (11.9\%), RA $(3.4 \%)$ and mixed cryoglobulinaemia $(2.3 \%)$. Numerous other studies have described the prevalence of these conditions among patients with CHC overall but not in relation to the presence of auto-abs. ${ }^{681532-38}$ To our knowledge, only four studies specifically investigated whether extrahepatic manifestations were more common among auto-ab-positive patients: three studies found no difference regarding mixed cryoglobulinaemia ${ }^{914} 16$ and one study found no difference regarding DM. ${ }^{14}$ One large study did find that certain extrahepatic manifestations were more common among those with either ANA or ASMA positivity, including vasculitis, abnormal thyroid function (ANA only), abnormal creatinine (ASMA only) and hypertension (ANA only). ${ }^{7}$ Our findings argue that the proclivity to developing auto-abs in CHC does not represent an underlying predisposition to extrahepatic manifestations in general.
Limitations of our study include its retrospective chart review design, which at times included data from handwritten charts. Some patients were therefore categorised as having a diagnosis or laboratory result based on mention in these handwritten notes. However, our primary outcomes are objective such as cirrhosis and HCC and based on clinical evaluation at the point of care, limiting the role a retrospective design may have on our findings. Furthermore, the use of the National Death Index allowed for a more comprehensive and complete follow-up that reinforces our data. Bias may also have been introduced because not all our patients with $\mathrm{CHC}$ received auto-ab testing and not every patient received testing for each of the queried auto-abs. Selection bias could have occurred regarding the decision to test for auto-abs in our HCV cohort and that the tested patients may have represented a subset of patients that were more ill-appearing to treating physicians. However, due to the retrospective design of this study, we were not able to ascertain the reasons for auto-abs testing in our cohort. We also conducted our study at a tertiary care centre. This may predispose our cohort to containing slightly more severe disease or different disease characteristics than the general population, but this observation holds true for both of our studied populations (being auto-ab positive or negative).

Strengths of our study include the ethnic diversity, overall number of patients, number of patients with positive auto-abs and the wide distribution of titres and patterns. A long enrolment and follow-up time, in addition to use of the National Death Index, were also strengths, allowing for a thorough analysis of the incidence of our primary outcomes.

In summary, in our cohort we found that auto-ab positivity was widely prevalent, was more commonly found in women, was not associated with increased prevalence of extrahepatic manifestation and had no impact on the natural history of CHC, except in that there was a lower chance of achieving SVR in those who received IFN-based regimens. This in turn did predict worse outcomes. SVR to IFN-free DAA therapy appeared similar in auto-ab-positive and auto-ab-negative patients. We postulate that auto-abs should not be routinely checked in patients with $\mathrm{CHC}$, unless there are other indications for workup of autoimmune liver disease.

Contributors AJG: study design, data collection, data analysis and interpretation, drafting of the manuscript. AKL: study design, data analysis and interpretation, data collection and critical review of the manuscript. CZ, JH, LAY and SCW: data collection, interpretation of data and critical review of the manuscript. JMV: data interpretation and critical review and revision of the manuscript. MHN: concept development, study design, data collection, data analysis and interpretation, drafting of the manuscript, critical revision of the manuscript and supervision of the study; guarantor of article. All authors approved the final version of the manuscript.

Funding This research received no specific grant from any funding agency in the public, commercial or not-for-profit sectors.

Competing interests MHN has served as a consultant for Bristol-Myers Squibb, Novartis, Intercept Pharmaceuticals, Anylam Pharmaceutical, Gilead Sciences, Janssen Pharmaceuticals, and Dynavax Laboratories and has received grant/ research support from Bristol-Myers Squibb, Gilead Sciences and Janssen 
Pharmaceuticals. JMV has research grant support from AbbVie, Gilead, Merck, Novartis, Sundise and Taiwan J. He participates in scientific advisory boards for AbbVie, Gilead, Merck, Novartis and Sundise.

Patient consent Not required.

Ethics approval Institutional Review Board of Stanford University.

Provenance and peer review Not commissioned; externally peer reviewed.

Data sharing statement Additional data available upon request: baseline laboratory analyses, Kaplan-Meier curves for high-titre comparisons and multivariate analyses for high-titre comparisons. Please direct inquiries to MHN via email.

Open Access This is an Open Access article distributed in accordance with the Creative Commons Attribution Non Commercial (CC BY-NC 4.0) license, which permits others to distribute, remix, adapt, build upon this work non-commercially, and license their derivative works on different terms, provided the original work is properly cited and the use is non-commercial. See: http://creativecommons.org/ licenses/by-nc/4.0/

(c) Article author(s) (or their employer(s) unless otherwise stated in the text of the article) 2018. All rights reserved. No commercial use is permitted unless otherwise expressly granted.

\section{REFERENCES}

1. Shire NJ, Sherman KE. Epidemiology of hepatitis $C$ virus: a battle on new frontiers. Gastroenterol Clin North Am 2015;44:699-716.

2. Blach S, Zeuzem S, Manns M, et al. Global prevalence and genotype distribution of hepatitis $C$ virus infection in 2015: a modelling study. Lancet Gastroenterol Hepatol 2017;2:161-76.

3. Saadoun D, Sadallah S, Trendelenburg M, et al. Anti-C1q antibodies in hepatitis C virus infection. Clin Exp Immunol 2006;145:308-12.

4. Cacoub P, Musset L, Amoura Z, et al. Anticardiolipin, anti-beta2glycoprotein I, and antinucleosome antibodies in hepatitis $C$ virus infection and mixed cryoglobulinemia. Multivirc Group. J Rheumatol 1997;24:2139-44.

5. Cacoub P, Ghillani P, Revelen R, et al. Anti-endothelial cell autoantibodies in hepatitis $\mathrm{C}$ virus mixed cryoglobulinemia. J Hepato 1999;31:598-603.

6. Cacoub P, Renou C, Rosenthal E, et al. Extrahepatic manifestations associated with hepatitis $C$ virus infection. Medicine 2000:79:47-56.

7. Cacoub P, Poynard T, Ghillani P, et al. Extrahepatic manifestations of chronic hepatitis C. Arthritis \& Rheumatism 1999;42:2204-12.

8. Clifford BD, Donahue D, Smith L, et al. High prevalence of serological markers of autoimmunity in patients with chronic hepatitis C. Hepatology 1995;21:613-9.

9. Luo JC, Hwang SJ, Li CP, et al. Clinical significance of serum autoantibodies in Chinese patients with chronic hepatitis C: negative role of serum viral titre and genotype. J Gastroenterol Hepatol 1998;13:475-9.

10. Cassani F, Cataleta M, Valentini $P$, et al. Serum autoantibodies in chronic hepatitis C: comparison with autoimmune hepatitis and impact on the disease profile. Hepatology 1997;26:561-6.

11. Chen $\mathrm{CH}$, Lee $\mathrm{CM}$, Chen $\mathrm{CH}$, et al. Prevalence and clinical relevance of serum autoantibodies in patients with chronic hepatitis C. Chang Gung Med J 2010;33:258-65.

12. Drygiannakis D, Lionis C, Drygiannakis I, et al. Low prevalence of liver-kidney microsomal autoantibodies of type 1 (LKM1) in hepatitis C seropositive subjects on Crete, Greece. BMC Gastroenterol 2001:1:4

13. Muratori P, Muratori L, Guidi M, et al. Clinical impact of non-organspecific autoantibodies on the response to combined antiviral treatment in patients with hepatitis C. Clin Infect Dis 2005;40:501-7.

14. Narciso-Schiavon JL, Freire FC, Suarez MM, et al. Antinuclear antibody positivity in patients with chronic hepatitis C: clinically relevant or an epiphenomenon? Eur J Gastroenterol Hepatol 2009;21:440-6.

15. Ozdemir FN, Turan M, Sezer S, et al. Prevalence of serological markers of autoimmunity in hemodialysis patients with hepatitis $\mathrm{C}$ virus. Transplant Proc 1998;30:848-9.

16. Peng YC, Hsieh SC, Yang DY, et al. Expression and clinical significance of antinuclear antibody in hepatitis $C$ virus infection. J Clin Gastroenterol 2001;33:402-6.
17. Stroffolini T, Colloredo G, Gaeta GB, et al. Does an 'autoimmune' profile affect the clinical profile of chronic hepatitis $C$ ? An Italian multicentre survey. J Viral Hepat 2004;11:257-62.

18. Williams MJ, Lawson A, Neal KR, et al. Autoantibodies in chronic hepatitis $C$ virus infection and their association with disease profile. $J$ Viral Hepat 2009;16:325-31.

19. Yee LJ, Kelleher P, Goldin RD, et al. Antinuclear antibodies (ANA) in chronic hepatitis $\mathrm{C}$ virus infection: correlates of positivity and clinical relevance. J Viral Hepat 2004;11:459-64.

20. Adeyemi OM, Attar B, Jensen D, et al. Serological markers of autoimmunity in patients infected with hepatitis $C$ virus: impact of HIV co-infection. HIV Med 2005;6:371-4.

21. Bai L, Lu HY, Feng ZR, et al. [Detection and the production mechanism of antinuclear antibodies (ANA) and anti-liver/kidney microsomal tpe 1 antibodies (anti-LKM1) in patients with chronic hepatitis C]. Zhonghua Shi Yan He Lin Chuang Bing Du Xue Za Zhi 2009;23:278-81.

22. Hsieh MY, Dai CY, Lee LP, et al. Antinuclear antibody is associated with a more advanced fibrosis and lower RNA levels of hepatitis $\mathrm{C}$ virus in patients with chronic hepatitis C. J Clin Pathol 2008;61:333-7.

23. Khairy M, El-Raziky M, El-Akel W, et al. Serum autoantibodies positivity prevalence in patients with chronic HCV and impact on pegylated interferon and ribavirin treatment response. Liver Int 2013;33:n/a-9.

24. Richardet JP, Lons T, Johanet C, et al. [Prevalence and characteristics of anti-tissue antibodies in chronic hepatitis caused by hepatitis C virus]. Gastroenterol Clin Biol 1994;18:819-23.

25. Mauss S, Berger F, Schober A, et al. Screening for autoantibodies in chronic hepatitis $\mathrm{C}$ patients has no effect on treatment initiation or outcome. J Viral Hepat 2013;20:e72-e77.

26. Hsieh MY, Dai CY, Lee LP, et al. Antinuclear antibody titer and treatment response to peginterferon plus ribavirin for chronic hepatitis C patients. Kaohsiung J Med Sci 2012;28:86-93.

27. Pérez R, Pravia R, Artímez ML, et al. A comparison between two induction regimes for the interferon treatment of chronic hepatitis $\mathrm{C}$. Response related factors. Rev Esp Enferm Dig 1997;89:159-73.

28. Bayraktar Y, Bayraktar M, Gurakar A, et al. A comparison of the prevalence of autoantibodies in individuals with chronic hepatitis $C$ and those with autoimmune hepatitis: the role of interferon in the development of autoimmune diseases. Hepatogastroenterology 1997;44:417-25

29. Bruix J, Sherman M. American association for the study of liver D. Management of hepatocellular carcinoma: an update. Hepatology 2011;53:1020-2.

30. Gregorio GV, Choudhuri K, Ma Y, et al. Mimicry between the hepatitis $\mathrm{C}$ virus polyprotein and antigenic targets of nuclear and smooth muscle antibodies in chronic hepatitis $\mathrm{C}$ virus infection. Clin Exp Immunol 2003;133:404-13.

31. Lian M, Hua J, Sheng L, et al. Prevalence and significance of autoantibodies in patients with alcoholic liver disease. J Dig Dis 2013;14:396-401.

32. Brito-Zerón P, Gheitasi H, Retamozo S, et al. How hepatitis C virus modifies the immunological profile of Sjögren syndrome: analysis of 783 patients. Arthritis Res Ther 2015;17:250.

33. Czaja AJ. Extrahepatic immunologic features of chronic viral hepatitis. Dig Dis 1997;15:125-44.

34. De Rosa FG, Pucillo LP, Coviello R, et al. Influence of age and autoimmunity on liver disease in HCV-associated type II mixed cryoglobulinemia. Hum Immunol 2002;63:751-7.

35. De Vita S, Damato R, De Marchi G, et al. True primary Sjögren's syndrome in a subset of patients with hepatitis $C$ infection: a model linking chronic infection to chronic sialadenitis. Isr Med Assoc J 2002;4:1101-5.

36. Ramos-Casals M, Muñoz S, Medina F, et al. Systemic autoimmune diseases in patients with hepatitis $C$ virus infection: characterization of 1020 cases (The HISPAMEC Registry). J Rheumatol 2009;36:1442-8.

37. Younossi Z, Park H, Henry L, et al. Extrahepatic manifestations of hepatitis C: a meta-analysis of prevalence, quality of life, and economic burden. Gastroenterology 2016;150:1599-608.

38. Sène D, Ghillani-Dalbin P, Limal N, et al. Anti-cyclic citrullinated peptide antibodies in hepatitis $C$ virus associated rheumatological manifestations and Sjogren's syndrome. Ann Rheum Dis 2006;65:394-7. 